\title{
Plasma CD14 decreases monocyte responses to LPS by transferring cell-bound LPS to plasma lipoproteins
}

\author{
Richard L. Kitchens, ${ }^{1}$ Patricia A. Thompson, ${ }^{1}$ Suganya Viriyakosol, ${ }^{2}$ Grant E. O’Keefe, ${ }^{3}$ \\ and Robert S. Munford 1,4
}

${ }^{1}$ Department of Internal Medicine, University of Texas Southwestern Medical Center, Dallas, Texas, USA

${ }^{2}$ Department of Pathology, University of California San Diego School of Medicine, Veterans Administration

San Diego Healthcare System, San Diego, California, USA

${ }^{3}$ Department of Surgery, and

${ }^{4}$ Department of Microbiology, University of Texas Southwestern Medical Center, Dallas, Texas, USA

Address correspondence to: Richard L. Kitchens, Department of Internal Medicine, University of Texas

Southwestern Medical Center, 5323 Harry Hines Boulevard, Dallas, Texas 75390-9113, USA.

Phone: (214) 648-6479; Fax: (214) 648-9478; E-mail: richard.kitchens@UTSouthwestern.edu.

Received for publication April 26, 2001, and accepted in revised form June 26, 2001.

\begin{abstract}
CD14, a myeloid cell-surface receptor and soluble plasma protein, binds LPS and other microbial molecules and initiates the innate immune response to bacterial invasion. The blood concentration of soluble CD14 (sCD14) increases during the systemic response to infection. Although high sCD14 blood levels have correlated with increased risk of dying from severe sepsis, sCD14 can diminish cell responses to LPS. We show here that in human serum, sCD14 increases the rate at which cell-bound LPS is released from the monocyte surface and binds to plasma lipoproteins. This enhanced rate of LPS efflux is associated with a significant reduction in the ability of monocytes to produce cytokines in response to LPS. Serum from septic patients reduced the LPS-monocyte interaction by as much as tenfold, and depletion of sCD14 from the serum restored LPS-monocyte binding and release kinetics to near normal levels. In serum from septic patients, monocyte-bound LPS also moved more rapidly into lipoproteins, which completely neutralized the biologic activity of the LPS that bound to them. In human plasma, sCD14 thus diminishes monocyte responses to LPS by transferring cellbound LPS to lipoproteins. Stress-related increases in plasma sCD14 levels may help prevent inflammatory responses within the blood.
\end{abstract}

J. Clin. Invest. 108:485-493 (2001). DOI:10.1172/JCI200113139.

\section{Introduction}

LPS (endotoxin) is one of the most potent of the known bacterial signal molecules. Sensitive detection of its presence provides animals with an early warning of bacterial invasion and mobilizes the immune response. In many individuals, however, an exaggerated response to endotoxin can be harmful, leading to severe sepsis, organ failure, and death. Plasma LPS binding protein (LBP) and the LPS binding receptor, membrane CD14, orchestrate sensitive responses to LPS (1) by facilitating its interaction with the LPS signaling receptor, Toll-like receptor 4 (TLR4; ref. 2), which is expressed on phagocytes and other LPS-responsive cells. The extreme sensitivity of the LPS recognition mechanism and the potentially damaging consequences of the ensuing host reaction underscore the need for effective mechanisms for controlling cell responses to LPS and removing it from the circulation and tissues.

Important factors in blood limit the interaction of LPS with circulating leukocytes. When pure LPS or bacterial outer membrane fragments are injected into the bloodstream, a large fraction of the LPS is cleared by the liver within 10 minutes (3-5), whereas most of the remaining LPS binds rapidly to lipoproteins, which neutralize its biologic activity $(6,7)$. LBP can transfer puri- fied LPS to HDL particles (8); we recently found that LBP can also extract native LPS from bacterial membranes and transfer it to HDL (9). Numerous in vitro and in vivo studies have shown that HDL and other plasma lipoproteins play a major role in decreasing LPSinduced host responses and lethality $(7,10,11)$.

LPS binding and recognition by leukocytes and LPS sequestration by lipoproteins were previously thought to be independent and essentially irreversible pathways of LPS traffic within the circulation. However, we recently found evidence that LPS can move from leukocyte surfaces to plasma lipoproteins and that this efflux of LPS decreases cell responses (12). We have now asked how the LPS-monocyte interaction is regulated within the blood during human responses to infection. We report that in the presence of undiluted plasma, LPS associates only transiently with monocytes and that the extent and duration of the LPS-cell interaction are regulated largely by membrane CD14 (mCD14). Sepsisinduced increases in plasma sCD14 can dramatically limit the amount of LPS that remains bound to monocytes and substantially reduce cytokine responses. We suggest that SCD14-mediated LPS efflux from cells is a previously unrecognized mechanism for controlling reactions to LPS within the bloodstream. 


\section{Methods}

Cells and reagents. Human monocytic THP-1 cells were transformed with human CD14 cDNA and cultured as described previously (13). PBMCs were separated from heparinized blood of normal human donors by centrifugation over Histopaque 1077 (Sigma Chemical Co., St. Louis, Missouri, USA). Recombinant human LPS binding protein (rLBP) was obtained from P. Tobias (The Scripps Research Institute, La Jolla, California, USA) (13). RPMI 1640, Cellgro Complete serum-free medium, and G418 were from Mediatech (Herndon, Virginia, USA). Other reagents were from Sigma Chemical Co. unless otherwise indicated.

sCD14 preparation. Recombinant human sCD14 (rsCD14) was generated in baculovirus-infected Hi-5 cells. rsCD14 with a C-terminal 6-histidine tag was purified on nickel-NTA-agarose and dialyzed against PBS (14). The sCD14-TEV-Fc fusion protein was made by cloning the coding sequence for the Fc fragment of human IgG by PCR from the plasmid PVL1392:Fc (a gift from C.F. Ware, La Jolla Institute for Allergy and Immunology, San Diego, California, USA). The oligonucleotides corresponding to the cleavage site for the Tobacco Etch Virus (TEV) protease (Life Technologies Inc., Rockville, Maryland, USA) were synthesized by Operon Technologies (Alameda, California, USA). The TEV and Fc coding sequences were inserted into the plasmid PfastbacI containing a gene for the 323 amino acids of human CD14. The resulting plasmid was used to make recombinant baculovirus as described in the Bac-to-Bac protocol (Life Technologies Inc.). The protein made in Hi-5 cells was purified on a protein-G column (Amersham Pharmacia Biotech, Piscataway, New Jersey, USA) and dialyzed against PBS. rsCD14 without epitope tags was prepared by digesting rsCD14-TEV-IgFc with TEV protease after the protease reagent mixtures had been treated with END-X (Associates of Cape Cod, Falmouth, Massachusetts, USA) to remove residual endotoxin. Control digests were prepared by omitting rsCD14.

Experimental subjects and serum preparation. Peripheral venous blood was drawn from normal volunteers and from patients who had experienced blunt trauma or burns, most of whom had developed infections as a result of their injuries. We analyzed 27 serum samples from these patients: 11 samples from nine trauma patients and 16 samples from 11 burn patients. Clinical classifications were made according to established criteria (15). All the patients met the clinical criteria for one or more stages of systemic inflammatory response syndrome (SIRS). Eight samples were from patients who had no evidence of infection at 1-4 days after injury (i.e., SIRS). The other 19 samples were from patients who had become infected at 3-46 days after injury; six were classified as sepsis, and 13 were classified as severe sepsis. Three of the patients classified as severe sepsis had septic shock. The protocol was approved by the Institutional Review Board of The University of Texas Southwestern Medical Center. For convenience, the term "sepsis serum" is used to refer to serum obtained from patients with sepsis or severe sepsis. Serum aliquots were stored at $-70^{\circ} \mathrm{C}$. Freezing did not alter the ability of the serum to promote release of cell-bound LPS.

LPS preparation, cell binding, and release. Escherichia coli LCD25 $\left(\left[{ }^{3} \mathrm{H}\right]\right.$ LPS $\left.\left[1.5 \times 10^{6} \mathrm{dpm} / \mu \mathrm{gg}\right]\right)$ was biosynthetically labeled and isolated as described elsewhere (16). Autoradiographs of Western blots of the $\left[{ }^{3} \mathrm{H}\right] \mathrm{LPS}$ preparation show that the radioactivity migrated with LPS on the gel. $\left[{ }^{3} \mathrm{H}\right]$ LPS binding assays were performed as described (17) by suspending $3.5 \times 10^{5}$ CD14-transfected THP- 1 cells or $2.5 \times 10^{6}$ PBMCs in $45 \mu \mathrm{l}$ of serum buffered with $10 \mathrm{mM}$ HEPES ( $\mathrm{pH}$ 7.4) in microcentrifuge tubes. The binding reaction was begun by adding $5 \mu$ l of partially disaggregated $\left[{ }^{3} \mathrm{H}\right] \operatorname{LPS}(13)(50$ $\mathrm{ng} / \mathrm{ml}$ final concentration) and incubating at $37^{\circ} \mathrm{C}$ with occasional mixing to keep the cells in suspension. Total cell-associated $\left[{ }^{3} \mathrm{H}\right] \mathrm{LPS}$ and protease-sensitive $\left[{ }^{3} \mathrm{H}\right]$ LPS on the cell-surface were measured (13). $\left[{ }^{3} \mathrm{H}\right]$ LPS release assays were performed as described previously (12). Briefly, $\left[{ }^{3} \mathrm{H}\right] \mathrm{LPS}$ was bound to the cells by incubating them for 5 minutes at $37^{\circ} \mathrm{C}$ with $\left[{ }^{3} \mathrm{H}\right] \mathrm{LPS}-$ sCD14 complexes (50 ng LPS per milliliter); this enables transfer of LPS to mCD14. The unbound complexes were washed away, the $\left[{ }^{3} \mathrm{H}\right]$ LPS-loaded cells were incubated in serum for the indicated times, and the cell-associated $\left[{ }^{3} \mathrm{H}\right]$ LPS and released $\left[{ }^{3} \mathrm{H}\right]$ LPS were measured after centrifugation to pellet the cells.

Peptide IV (0.6 $\mathrm{mM})$, a peptide that binds and inactivates complement factor C3 (18), was added to the serum samples 10 minutes before the assay was begun. This treatment prevented the cell loss and membrane permeability that were observed when THP-1 cells were incubated in many of the serum samples from injured or infected patients. After each LPS binding or release assay, the cells were counted and membrane permeability was measured by trypan blue exclusion (12). No significant cell loss was observed, and cell viability was $90 \%$ or greater. Peptide IV did not alter LPS binding or release from the cells; similar results were obtained in these assays when $10 \mathrm{mM}$ EDTA replaced Peptide IV as a complement inhibitor.

Lipoprotein isolation and analysis. The total lipoprotein fraction (LP) of serum was isolated by ultracentrifugal flotation in $\mathrm{KBr}(d<1.21 \mathrm{~g} / \mathrm{ml})(12)$. Free LPS and LPSsCD14 complexes did not float with LPS-lipoprotein complexes. In some experiments, the lipoproteins were concentrated by recentrifugation and dialyzed against cold $0.9 \% \mathrm{NaCl}$ containing 0.25 mM EDTA ( $\mathrm{pH} 8.0$ ). $\left[{ }^{3} \mathrm{H}\right]$ LPS in the $\mathrm{KBr}$ fractions was measured by liquid scintillation counting as described previously (12).

Total (esterified and unesterified) cholesterol and choline-containing phospholipids were measured using kits from Sigma Chemical Co. and Wako Chemicals USA Inc. (Richmond, Virginia, USA), respectively. HDL cholesterol and phospholipids were measured by precipitating the LDLs (e.g., LDL and VLDL) with phosphotungstic acid reagent (Paramax HDL Cholesterol Precipitating Reagent; Dade International Inc., 
Miami, Florida, USA) according to the manufacturer's instructions. HDL levels were measured in the supernatants, and non-HDL (LDL/VLDL) lipoprotein levels were calculated by subtracting the HDL levels from those of the unfractionated serum.

Immunodepletion of $s C D 14$. sCD14-depleted serum was prepared using endotoxin-free reagents. Anti-CD14 $\mathrm{mAb} 60 \mathrm{bca}$ was immobilized on agarose beads by covalent attachment of the protein's carbohydrate residues using the Carbolink Kit (Pierce Chemical Co., Rockford, Illinois, USA). Control beads were prepared by treatment with formaldehyde followed by washing with PBS containing $0.1 \mathrm{M}$ glycine. Before exposure to serum, the beads were washed with five bed volumes of pyrogen-free PBS. The undiluted serum was passed over $60 \mathrm{bca}$ beads or control beads four times in a small column. Typically, $30 \mu \mathrm{l}$ of $60 \mathrm{bca}$ beads was required to deplete $1 \mathrm{ml}$ of normal serum. This treatment did not deplete LBP from the serum; the resulting LBP concentrations measured by ELISA were $86 \% \pm 12 \%$ (mean $\pm \mathrm{SD}, n=5$ ) of precolumn levels, and the LBP levels in paired SCD14-depleted and control samples did not differ by more than $10 \%$ from each other. ELISA assays for mouse IgG in the depleted serum confirmed that the CD14 mAb was not released from the beads and thus could not bind to mCD14 when the depleted serum was mixed with monocytes; we found less than $10 \mathrm{ng} / \mathrm{ml}$ mouse IgG in the treated serum, less than the amount of residual sCD14 in the samples. In some experiments as noted, sCD14 was depleted from serum using $60 \mathrm{bca}$ or a nonimmune control antibody attached to magnetic beads as described previously (12).

Cell stimulation and ELISA assays. To measure the stimulatory potency of LPS-lipoprotein complexes, adherent normal human monocytes were incubated with free $\left[{ }^{3} \mathrm{H}\right]$ LPS or dialyzed $\left[{ }^{3} \mathrm{H}\right]$ LPS-lipoprotein complexes in $1 \mathrm{ml}$ RPMI 1640 containing heat-inactivated serum (10\% FCS and $1 \%$ human serum) (for 6 hours at $37^{\circ} \mathrm{C}$ in $\left.5 \% \mathrm{CO}_{2}\right)$. TNF- $\alpha$ and IL- 6 were measured in the culture supernatants by ELISA.

The effects of rsCD14 on the responses of LPS-loaded PBMCs were determined as follows. Normal human PBMCs $\left(50 \times 10^{6} \mathrm{cells} / \mathrm{ml}\right)$ were incubated with [ $\left.{ }^{3} \mathrm{H}\right]$ LPSsCD14 complexes (13) $(0.3 \mathrm{ng}$ LPS/ml) for 5 minutes at $37^{\circ} \mathrm{C}$ in RPMI 1640 containing 20 mM HEPES ( $\mathrm{pH} 7.4$ ) and $0.1 \mathrm{mg} / \mathrm{ml}$ low-endotoxin BSA. The cells were pelleted (for 2 minutes at $750 \mathrm{~g}$ ) and washed three times with RPMI 1640. The LPS-loaded cells $\left(4 \times 10^{5}\right.$ PBMCs in $10 \mu \mathrm{l}$ ) were added to $190 \mu \mathrm{l}$ of undiluted autologous heparinized whole blood containing $0-4 \mu \mathrm{g} / \mathrm{ml} \mathrm{rsCD} 14$ in sterile $12 \times 75 \mathrm{~mm}$ polycarbonate tubes. The mixtures were incubated (for 5 hours at $37^{\circ} \mathrm{C}$ in $5 \% \mathrm{CO}_{2}$ ). A total of $600 \mu \mathrm{l}$ of cold PBS was added; the cells were removed by centrifugation; and TNF- $\alpha$, IL- $1 \beta$, and IL- 6 were measured in the supernatants by ELISA. Under these conditions, the average cytokine levels in the absence of rsCD14 were $1.6 \mathrm{ng}$ TNF- $\alpha$ per milliliter, $2.0 \mathrm{ng}$ IL-1 $\beta$ per milliliter, and $6.1 \mathrm{ng}$ IL- 6 per milliliter in the undiluted blood, whereas levels of all of these cytokines in the absence of LPS were less than or equal to $0.02 \mathrm{ng} / \mathrm{ml}$. The total cell-associated $\left[{ }^{3} \mathrm{H}\right]$ LPS measured before adding the PBMCs to the blood indicated that the blood/PBMC mixture contained approximately 7 pg cell-associated LPS per milliliter. Given that this concentration of free LPS was insufficient to stimulate a significant response in whole blood, we assume that most or all of the cytokine response was derived from the LPS-loaded PBMCs, rather than from LPS transfer to naive monocytes in the blood. Response assays using free LPS were performed in the same manner except that the PBMCs were omitted and free LPS $(0.03 \mathrm{ng} / \mathrm{ml})$ was added to the undiluted blood. Stimulation of whole blood with recombinant human IL-1 $\beta$ (rIL-1 $\beta$; Becton Dickinson PharMingen, San Diego, California, USA) was performed by adding $10 \mathrm{ng}$ of rIL-1 $\beta$ per milliliter of blood and measuring IL- 6 .

The effects of immunodepletion of serum sCD14 on responses of LPS-loaded PBMCs were determined by adding LPS-loaded PBMCs (in $10 \mu \mathrm{l}$ ) to $190 \mu \mathrm{l}$ of control-treated or sCD14-depleted autologous serum. The mixtures were incubated and analyzed as described in the whole-blood assay above. Response assays using free LPS were performed in the same manner except that LPS-free PBMCs were added to the serum followed by free LPS $(0.03 \mathrm{ng} / \mathrm{ml})$.

The LBP ELISA reagents and method were from P. Tobias (12). Other serum proteins were measured by commercial ELISA kits (the sCD14 kit was from R\&D Systems Inc., Minneapolis, Minneapolis, USA; the TNF- $\alpha$, IL-1 $\beta$, and IL-6 kits were from BD PharMingen).

Statistics. ANOVA using Tukey's multiple comparison test was performed using Prism 3.0 (Graph Pad Software Inc., San Diego, California, USA). Pearson correlation coefficient $(r)$ for XY pairs were also computed by Prism 3.0.

\section{Results}

LPS binds transiently to monocytes in the presence of undiluted serum. LPS interacts with monocytes and plasma lipoproteins along the pathways shown schematically

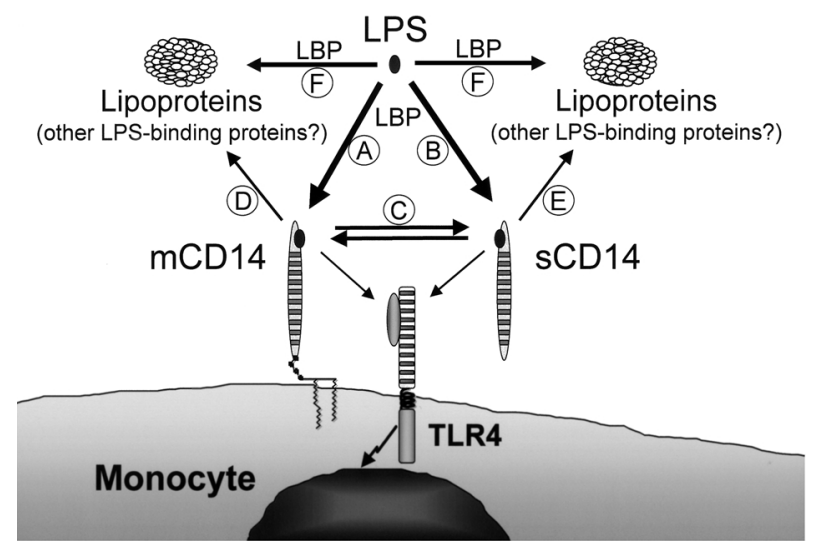

\section{Figure 1}

LPS binds transiently to monocytes in the presence of undiluted serum. See Results. 
a

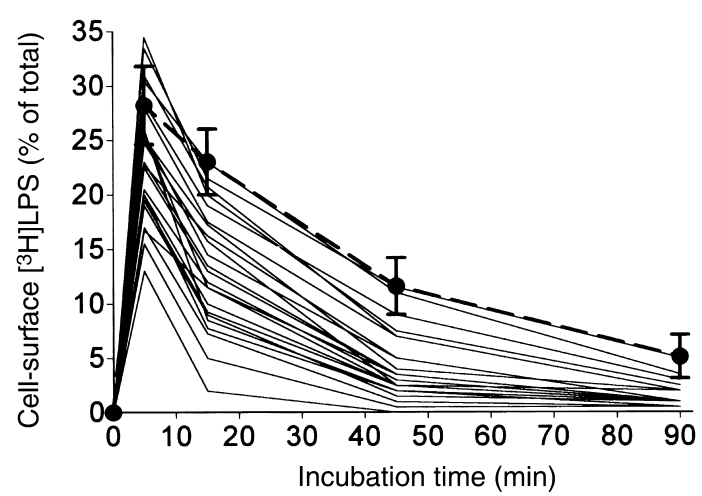

b

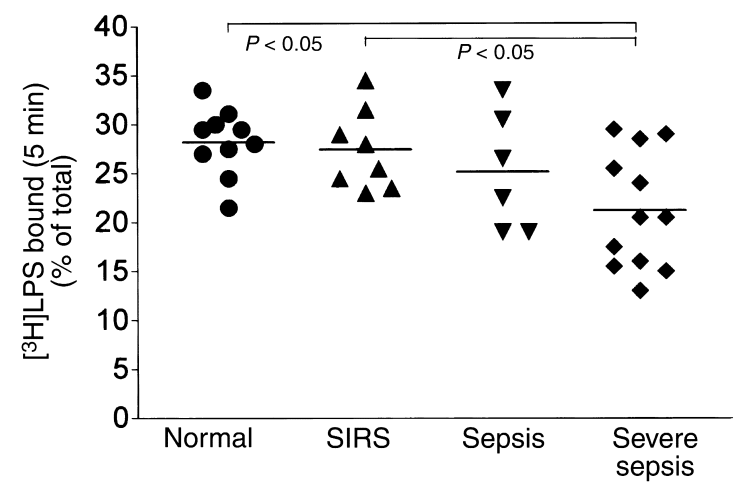

C

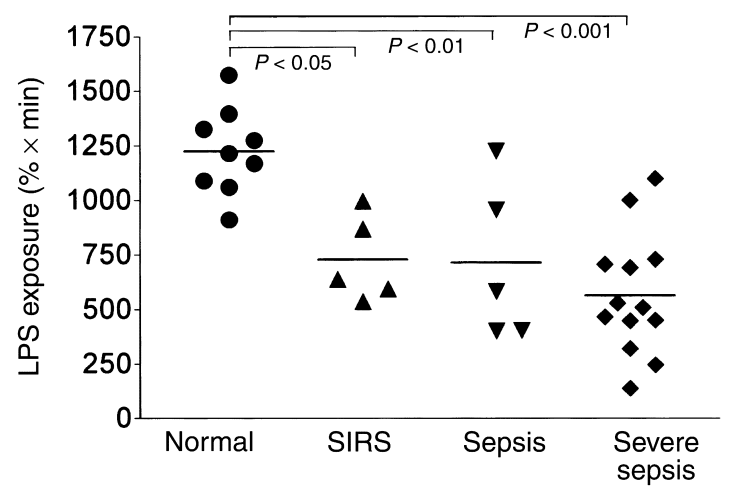

Figure 2

Sepsis serum decreases LPS binding to monocytes. CD14-transfected THP-1 cells were suspended in undiluted human serum, onetenth volume of $\left[{ }^{3} \mathrm{H}\right]$ LPS was added $(50 \mathrm{ng} / \mathrm{ml}$ final), and the mixtures were incubated for the indicated times. Cell-associated LPS was measured as percent of the total added $\left[{ }^{3} \mathrm{H}\right]$ LPS. Total LPS (data not shown), protease-sensitive (cell-surface) (data shown), and protease-resistant (internalized) LPS (data not shown) were measured. (a) Data from normal donors (means \pm SD of 15 determinations from nine individuals) are shown with filled circles and dashed line. Data from patients with trauma, burns, or sepsis (23 samples from 18 patients) are shown individually by solid lines without symbols as means of duplicate determinations; the ranges (data not shown) do not deviate more than $2 \%$ on the $y$-axis. (b) The amount of LPS that was bound to the cells at the 5-minute time point in normal serum (Normal) and patient serum with the indicated syndromes are shown. Solid lines denote means. $P$ values (ANOVA) are shown with brackets. (c) The total LPS exposure (area under each curve from 0 to 90 minutes) was computed from the data in a for each clinical group. in Figure 1. LBP mediates LPS binding to cells (Figure $1, \mathrm{~A}$; reviewed in ref. 1 ) and to plasma lipoproteins (Figure 1, F; ref. 9). In diluted serum, these mechanisms direct added LPS largely to mCD14 expressed on cells, whereas in undiluted serum, LPS binds only transiently to the cells (17) (Figure 2a). LPS-cell binding in the presence of LBP is rapid in either circumstance and proceeds by direct binding to $\mathrm{mCD} 14$ (Figure $1, \mathrm{~A}$ ) or indirectly via sCD14 (Figure 1, B and C). We recently found that there is also a mechanism for removing LPS from monocyte surfaces (12). Cell-bound LPS moves into plasma lipoproteins either directly from mCD14 (Figure $1, \mathrm{D}$ ) or indirectly via sCD14 (Figure $1, \mathrm{C}$ and $\mathrm{E}$ ) by mechanisms that do not involve LBP. LPS monomers can rapidly and reversibly move between mCD14 and $\mathrm{sCD} 14$ so that an equilibrium is established between the LPS that is bound to each form of CD14. The rate of LPS release from monocytes is greatly enhanced by undiluted serum or by rsCD14 if lipoproteins are present (12). Thus, the transient binding of LPS to monocytes in undiluted serum may be explained both (a) by the sequestration of free LPS by lipoproteins and other LPS-binding components of serum (Figure 1, F) and (b) by the release of cell-bound LPS (Figure 1, C-E). LPS binding to lipoproteins is essentially irreversible. In the experiments reported here, we studied the role of sCD14 in these processes and the impact of elevated sCD14 levels in serum derived from septic patients.

Sepsis serum decreases the amount and duration of LPS binding to monocytes. The fraction of cell-associated LPS that remains on the cell surface can be quantitated by virtue of its susceptibility to removal by proteinase $\mathrm{K}$ (13). The heavy dashed line in Figure 2a shows that when LPS was added to cells that were suspended in undiluted normal human serum, the amount of cell-surface $\left[{ }^{3} \mathrm{H}\right]$ LPS increased rapidly, peaked at approximately 5 minutes, and then slowly declined. After 90 minutes, the cell-bound LPS approached a plateau at which relatively little LPS remained on the cell surface. The continuous lines show comparable data from 23 serum samples from 18 patients. The data show that whereas LPS bound transiently to the cells in normal serum, the amount and duration of LPS binding were reduced much more in the patient serum.

To assess the LPS-monocyte interaction as a function of both time and the amount of LPS bound, we integrated the areas under the curves in Figure 2a. We refer to this measurement as the "total exposure" of cells to LPS. In patient serum, the total LPS exposure was as low as $11 \%$ of that measured in normal serum. The peak binding (5 minutes) and total exposure of the cells to LPS were correlated with the patients' clinical status in Figure 2, b and c. As shown in Figure 2b, the amount of LPS-cell binding observed in 5 minutes was decreased only in sera from patients with severe sepsis. The total exposure of the cells to LPS (Figure 2c), however, was significantly decreased in all clinical groups.

The cells internalized relatively small amounts of LPS. The protease-protected (presumably internalized) 
a

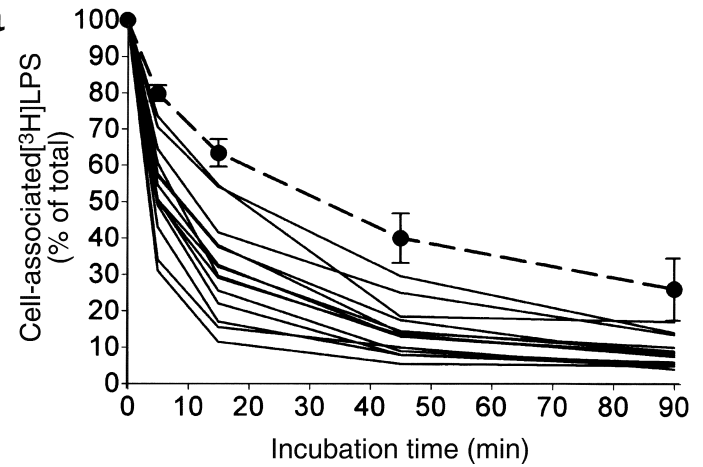

b

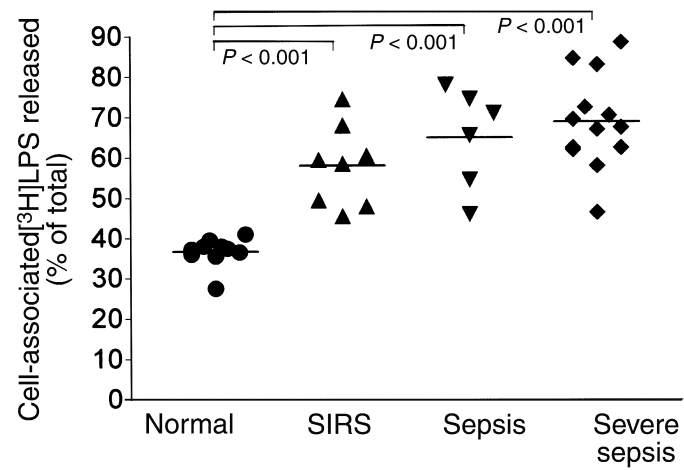

Figure 3

Sepsis serum accelerates the release of cell-bound LPS from monocytes. (a) Time courses of $\left[{ }^{3} \mathrm{H}\right]$ LPS release from THP-1 cells were measured in undiluted serum as described in Methods. The total cell-associated $\left[{ }^{3} \mathrm{H}\right]$ LPS is shown as the percentage of that initially bound to the cells. The data from normal serum are means \pm SD of 15 determinations from nine different normal donors (closed circles and dashed line). The data from the patients ( 14 samples from ten individuals) are shown individually by solid lines. The means of duplicate determinations are shown without symbols, and the ranges (data not shown) do not deviate more than $3 \%$ on the $y$-axis. (b) The analyses according to clinical groups were performed as in Figure $2 b$ using data from the normal samples and 27 patient samples including those shown in $\mathbf{a}$. The $y$-axis denotes the percentage of LPS released at the 15-minute time point.

$\left[{ }^{3} \mathrm{H}\right]$ LPS reach a maximum in 5 minutes and showed little or no change at later time points (data not shown) (17). The cells internalized less [ $\left.{ }^{3} \mathrm{H}\right]$ LPS in the patient serum; in normal serum, $7.6 \% \pm 4.0 \%$ (mean $\pm \mathrm{SD}$, $n=9$ ) of the total added $\left[{ }^{3} \mathrm{H}\right]$ LPS was internalized at the 90-minute time point, whereas in the patient serum, $4.9 \% \pm 2.4 \%(n=23)$ was internalized. The internalized fractions (90 minutes) of the total cell-associated LPS (5-minute peak) were $20 \%$ and $13 \%$ for normal and patient sera, respectively. Similar results were obtained whether plasma or serum was used. Results obtained using normal human monocytes were similar except that they appeared to internalize a larger fraction of the cell-associated $\left[{ }^{3} \mathrm{H}\right] \mathrm{LPS}$; in normal serum, $4-9 \%$ of the total added [ $\left.{ }^{3} \mathrm{H}\right]$ LPS $(26-36 \%$ of the 5 -minute peak cell-associated LPS) was internalized (two experiments; data not shown).
Because the total exposure of cells to LPS is a function of both LPS binding and the release of cell-bound LPS, we next measured the release of $\left[{ }^{3} \mathrm{H}\right] \mathrm{LPS}$ from cells that were preloaded with $\left[{ }^{3} \mathrm{H}\right] \mathrm{LPS}$ and then incubated in the same serum samples. As shown in Figure 3a, patient serum enhanced LPS release. In some cases, almost all of the cell-associated LPS was released within 15-45 minutes. The analysis of all 27 samples showed that LPS release was significantly increased in all clinical groups when measured at either 5 minutes (data not shown) or 15 minutes (Figure 3b). Differences between the normal and patient groups were not due to cell loss, cell permeability, or day-to-day variations in either the LPS binding or release assay. An aliquot of serum from the same normal individual was analyzed each time an assay was performed, and the results did not deviate from the normal ranges shown in Figures 2a and 3a. Loss of mCD14 from the cells does not appear to play a role in the rapid phase of LPS release from the cells, as flow cytometric analysis showed that $\mathrm{mCD} 14$ density was unchanged after incubating LPS-loaded THP-1 cells with normal $(n=1)$ or patient $(n=10)$ serum for 15 minutes (data not shown).

Sepsis serum accelerates the binding and neutralization of released LPS by plasma lipoproteins. To determine whether cell-associated LPS binds to lipoproteins in sepsis serum, we separated the total lipoproteins from the serum-containing supernatants of $\left[{ }^{3} \mathrm{H}\right]$ LPS-loaded cells by ultracentrifugal flotation (12). We found that in serum from septic patients, cell-associated LPS was both released from the cells more rapidly and bound to lipoproteins more rapidly than in normal serum. The percent of the total cell-associated $\left[{ }^{3} \mathrm{H}\right] \mathrm{LPS}$ released into the serum in 10 minutes was $73 \% \pm 10 \%$ (mean $\pm \mathrm{SD}, n=4$ patients) and $27 \% \pm 3 \%$ ( $n=6$ normal donors). The fractions of the released $\left[{ }^{3} \mathrm{H}\right]$ LPS that had bound to lipoproteins were $84 \% \pm 10 \%(n=4$ patients) and $62 \% \pm 4 \%(n=6$ normal donors). After 45 minutes, $88 \%$ and $82 \%$ of the released $\left[{ }^{3} \mathrm{H}\right] \mathrm{LPS}$ had bound to lipoproteins in patient and normal sera, respectively. These data were surprising in view of the fact that HDL, the major acceptor for cell-associated LPS in normal plasma (12), declines to low levels during sepsis $(19,20)$. Our measurements confirmed that both HDL cholesterol $(8 \pm 6 \mathrm{mg} / \mathrm{dL}$ [mean $\pm \mathrm{SD}$, $n=27])$ and HDL phospholipid levels $(36 \pm 12 \mathrm{mg} / \mathrm{dL})$ in the patient serum were lower than those in normal serum $(47 \pm 8 \mathrm{mg}$ cholesterol/dL and $101 \pm 16 \mathrm{mg}$ phospholipid/dL; $n=11)$.

To determine whether lipoproteins in the patients' sera can neutralize the stimulatory activity of LPS, we pooled serum samples from nine patients, incubated the serum with $\left[{ }^{3} \mathrm{H}\right] \mathrm{LPS}$, and isolated the LPS-lipoprotein complexes by ultracentrifugal flotation. We found that the LPS-lipoprotein complexes had almost no ability to stimulate IL- 6 or TNF- $\alpha$ release from normal human monocytes; LPS-lipoprotein complexes added at $50 \mathrm{ng}$ LPS per milliliter produced responses that were $5-10 \%$ of the maximal responses produced by 0.1 $\mathrm{ng} / \mathrm{ml}$ of free LPS (data not shown). Similar results 

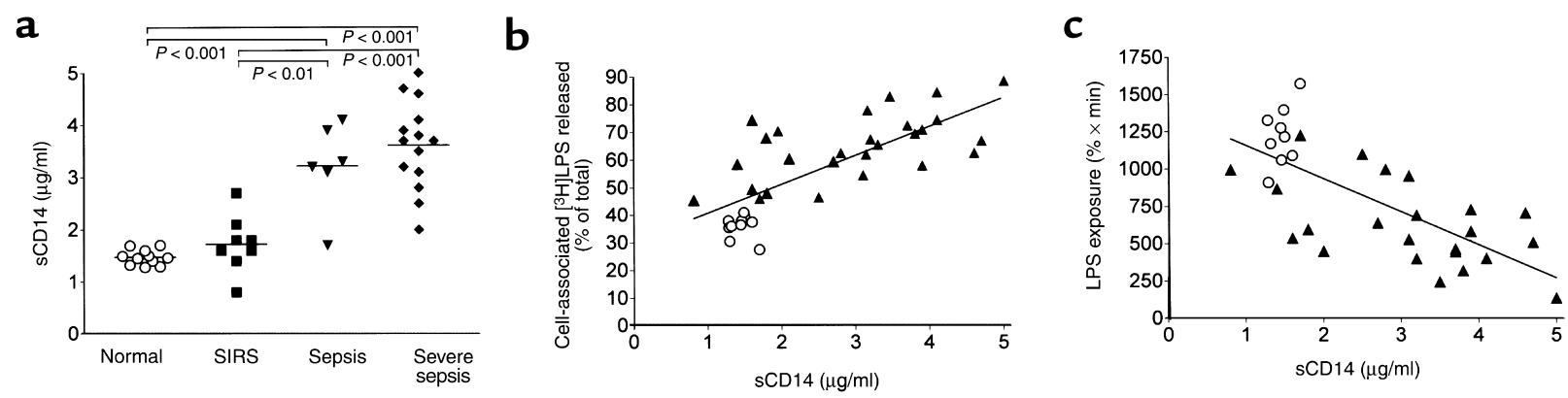

\begin{abstract}
Figure 4
Release of cell-bound LPS correlates with serum soluble CD14 levels. (a) Serum sCD14 levels were analyzed according to clinical groups as described in Figure $2 \mathrm{~b}$. (b) The percent of the total cell-associated $\left[{ }^{3} \mathrm{H}\right] \mathrm{LPS}$ released from THP-1 cells during a 15-minute incubation in undiluted serum derived from the individuals shown in a is plotted against their sCD14 levels. (c) Total exposure of the cells to [ ${ }^{3} \mathrm{H}$ ] LPS (see Figure $2 \mathrm{c}$ ) is plotted against SCD14. Patient serum (closed triangles) and normal serum (open circles) are shown in b and $\mathbf{c}$. Linear regression lines are shown for data in all groups.
\end{abstract}

were also obtained when LPS-lipoprotein complexes were isolated from individual samples from three patients; these LPS-lipoprotein complexes had no ability to stimulate TNF- $\alpha$ and IL-6 production in whole blood when added at concentrations that have maximal activity for free LPS (data not shown).

Release of cell-bound LPS correlates with serum soluble CD14 levels. Whereas SIRS patients without infection had normal or slightly elevated levels of sCD14 (Figure 4a), high levels of sCD14 were observed in serum from patients with sepsis or severe sepsis. When serum sCD14 concentrations were plotted against the percent of cell-associated $\left[{ }^{3} \mathrm{H}\right]$ LPS released during the first 15 minutes of exposure to serum (Figure $4 \mathrm{~b}$ ), linear regression analysis revealed a significant correlation $(r=0.7339, P<0.0001, n=40)$. Similar results were obtained when the sCD14 levels were plotted against the percentage of cell-associated LPS released in $5 \mathrm{~min}$ utes (data not shown). sCD14 levels correlated inversely with total LPS exposure (Figure 4c) $(r=0.7152$, $P<0.0001, n=32$ ) and with the amount of LPS that bound to the cells in 5 minutes (data not shown) $(r=0.7714, P<0.0001, n=36)$. We also found that other acute-phase proteins (SAA, LBP, and sPLA ${ }_{2}$ ) correlated significantly with LPS efflux and inversely with LPS binding and total exposure (data not shown).

Elevated levels of 5 CD14 decrease the LPS-monocyte interaction by enhancing LPS release. Having previously demonstrated that SCD14 promotes the release of cellbound LPS in normal plasma (12), we sought to determine the effect of the high sCD14 concentrations found in sepsis serum. Immunodepletion of sCD14 from normal serum (shown by dashed lines in Figure $5 a)$ increased the total exposure of cells to LPS; the amount of LPS bound increased somewhat, and the rate of LPS efflux decreased. Normal LPS exposure was restored by adding back rsCD14 to the depleted serum. sCD14 depletion produced a greater increase in LPS exposure in sepsis serum (Figure 5a, solid lines). Increasing the sCD14 concentration in normal control serum to the high levels seen in sepsis serum decreased exposure of cells to LPS (data not shown).

We also tested the effect of SCD14 depletion on the release of LPS from LPS-loaded cells as in Figure 3a. As

Table 1

Depletion of endogenous sCD14 in serum increases monocyte responses to LPSA

\begin{tabular}{llll}
\hline sCD14 & TNF- $\alpha$ & IL-1 $\beta$ & IL-6
\end{tabular}

LPS-loaded PBMCs

\begin{tabular}{|c|c|c|c|c|}
\hline Control & 1.51 & $100 \% \pm 2 \%(12)$ & $100 \% \pm 5 \%(8)$ & $100 \% \pm 5 \%(7)$ \\
\hline sCD14-depleted & 0.22 & $154 \%^{\mathrm{B}} \pm 19 \%(12)$ & $276 \%^{\mathrm{C}} \pm 24 \%(8)$ & $221 \%^{\mathrm{C}} \pm 21 \%(8)$ \\
\hline rsCD14 added & 1.35 & $94 \% \pm 9 \%(6)$ & $230 \% \pm 28 \%(5)$ & $136 \% \pm 20 \%(5)$ \\
\hline \multicolumn{5}{|l|}{ free LPS } \\
\hline Control & 1.51 & $100 \% \pm 3 \%(9)$ & $100 \% \pm 9 \%(9)$ & $100 \% \pm 14 \%(9)$ \\
\hline sCD14-depleted & 0.22 & $148 \%^{\mathrm{D}} \pm 13 \%(9)$ & $271 \%^{\mathrm{D}} \pm 52 \%(9)$ & $189 \%^{\mathrm{D}} \pm 24 \%(9)$ \\
\hline rsCD14 added & 1.35 & $77 \% \pm 9 \%(6)$ & $83 \% \pm 21 \%(6)$ & $118 \% \pm 19 \%(6)$ \\
\hline
\end{tabular}

${ }^{A}$ SCD14 levels were reduced by passing normal serum over agarose beads to which an anti-CD14 mAb was covalently bound. LPS-loaded autologous PBMC were incubated for 5 hours with undiluted serum that was passed over control beads (control), sCD14-depleted serum, and sCD14-depleted serum to which rsCD14 was added. Cytokines released into the serum were measured by ELISA. In other experiments, autologous PBMCs were mixed with free LPS (0.03 ng/ml) in serum that was prepared as described in the text. PBMC incubated in sera without LPS did not produce measurable levels of cytokines (data not shown). Mean \pm SEM is shown as percent of control for $(n)$ determinations in 3 (IL-1 $\beta$, IL- 6 ) or 4 (TNF- $\alpha$ ) separate experiments. ${ }^{B} P<0.05$, ${ }^{C} P<0.001$, and ${ }^{D} P<0.01$, reflecting significant differences between sCD14-depleted and Control sera determined by ANOVA. 
shown in Figure 5b, sCD14 depletion decreased LPS release from cells in both normal and sepsis/SIRS serum; this activity was restored by adding rsCD14. sCD14 levels $(\mathrm{mg} / \mathrm{ml})$ were $1.7,1.4,1.7$, and 1.5 in the normal control samples, 3.6, 2.1, 2.1, and 1.6 in the patients' control samples, and $\leq 0.02$ in the immunodepleted samples. sCD14-depleted sera from the sepsis and SIRS patients still possessed a greater ability to promote LPS release from cells than did CD14-depleted normal sera. These results suggest that one or more unidentified factors in the sepsis/SIRS serum can also enhance LPS release from cells.

We next increased sCD14 levels in normal serum by adding rsCD14 and measured $\left[{ }^{3} \mathrm{H}\right]$ LPS transfer from monocytes to serum lipoproteins during a 10-minute incubation. As shown in Figure 5c, the release of cellbound LPS increased with increasing sCD14 concentration, whereas the fraction of the released LPS that bound to lipoproteins remained constant (see legend to Figure 5). The data therefore suggest that increasing the sCD14 concentration increases the rate of transfer of LPS from cells to lipoproteins principally by increasing LPS release from cells.

Physiological concentrations of $s C D 14$ downregulate responses in LPS-loaded monocytes and to free LPS in blood. To determine whether concentrations of sCD14 equivalent to those observed in septic plasma could diminish responses in LPS-loaded cells, we incubated LPSloaded normal PBMCs in autologous whole blood to which was added increasing amounts of rsCD14. As shown in Figure 6, increasing the sCD14 concentration by only $1 \mu \mathrm{g} / \mathrm{ml}$ (endogenous + exogenous sCD $14=2.5$ $\mu \mathrm{g} / \mathrm{ml}$ ) produced a significant inhibitory effect. Inhi- bition appeared to be LPS specific, as the rsCD14 did not inhibit the ability of rIL- $1 \beta$ to stimulate IL- 6 production in whole blood (data not shown). Removing the C-terminal histidine tag from the rsCD14 did not diminish its inhibitory activity. rsCD14 also inhibited the ability of free LPS to stimulate responses in whole blood. Free LPS was added to whole blood (0.03-0.1 ng LPS per milliliter) that contained either 0 or $4 \mu \mathrm{g} / \mathrm{ml}$ rsCD14, and the mixtures were incubated and analyzed as described in Figure 6. The presence of the rsCD14 reduced the levels of IL- $1 \beta$ to $52 \% \pm 2 \%$ of control, IL- 6 to $47 \% \pm 6 \%$ of control, and TNF- $\alpha$ to $66 \% \pm 5 \%$ of control (mean \pm SEM of three experiments).

Likewise, reducing the endogenous SCD14 concentration of normal serum by immunodepletion increased the cytokine responses of monocytes that were incubated in the undiluted serum whether the monocytes were preloaded with LPS or incubated in serum containing free LPS (Table 1). Restoring normal sCD14 concentrations in the depleted sera by adding rsCD14 decreased the cell responses. These data suggest that even normal concentrations of sCD14 have an inhibitory effect on LPS-induced cytokine production in the blood.

\section{Discussion}

These studies produced three significant findings. First, whereas LPS binds transiently to monocytes in the presence of normal serum, the LPS-monocyte interaction is substantially decreased by factors in the serum of patients with SIRS, sepsis, and severe sepsis. Our data suggest that the amount and duration of the LPS-monocyte interaction decrease principally because of elevated a

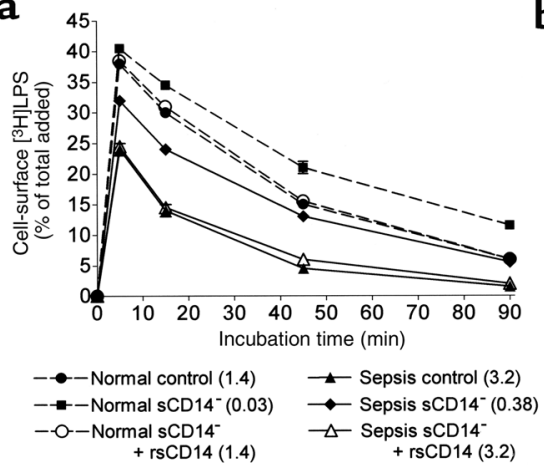

b

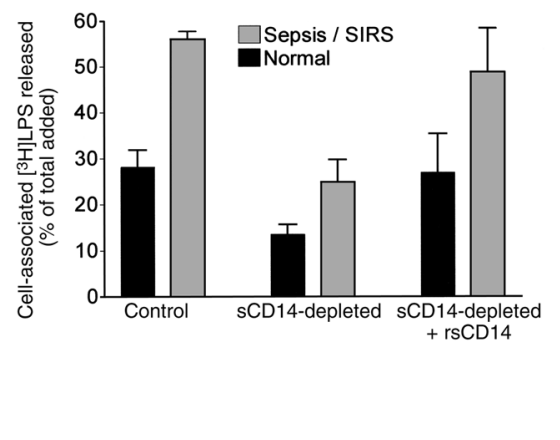

c

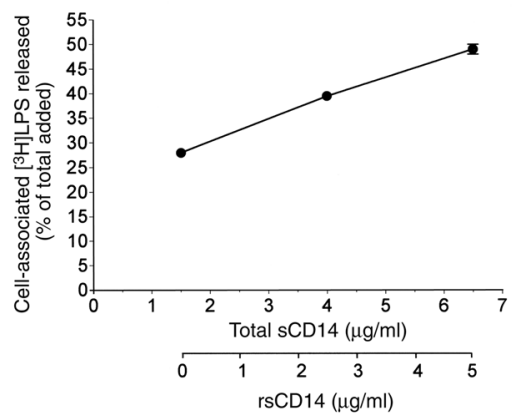

\section{Figure 5}

SCD14 modulates LPS binding to, and release from, monocytes. (a) Time course of $\left[{ }^{3} \mathrm{H}\right] \mathrm{LPS}$ binding to THP-1 cells in undiluted serum from a normal donor (dashed lines) or a patient with severe sepsis (solid lines) after immunodepleting the serum with anti-CD14-agarose (sCD14-), treating the serum with control beads (control), or adding rsCD14 back to sCD14-depleted serum (sCD14- + rsCD14). Numbers in parentheses are $\mathrm{sCD} 14$ concentrations in micrograms per milliliter. The experiment was repeated with similar results. (b) The release of $\left[{ }^{3} \mathrm{H}\right] \mathrm{LPS}$ from THP-1 cells was measured after incubating the cells for 15 minutes with undiluted normal or sepsis/SIRS serum that was immunodepleted of sCD14 using anti-CD14-magnetic beads (sCD14-depleted) or treated with nonimmune control antibody-magnetic beads (control) as described (12). sCD14 concentrations were restored to their original levels by adding rsCD14 to the sCD14-depleted sera (sCD14-depleted + rsCD14). Means \pm SD of four experiments are shown. Serum from four normal donors, two severe sepsis patients, and two SIRS patients was analyzed. (c) The release of $\left[{ }^{3} \mathrm{H}\right]$ LPS from THP- 1 cells was measured after incubation for 10 minutes at $37^{\circ} \mathrm{C}$ in undiluted normal serum containing increasing concentrations of rsCD14. The endogenous serum sCD14 was $1.5 \mu \mathrm{g} / \mathrm{ml}$. The percentages of the released [3H] LPS that was bound to lipoproteins in the total lipoprotein fraction $(d<1.21 \mathrm{~g} / \mathrm{ml}$ ) were $67 \% \pm 1 \%, 68 \% \pm 1 \%$, and $66 \% \pm 1 \%$ (mean \pm range of duplicate determinations) at $\mathrm{sCD} 14$ concentrations of $1.5,4$, and $6.5 \mu \mathrm{g} / \mathrm{ml}$, respectively. 


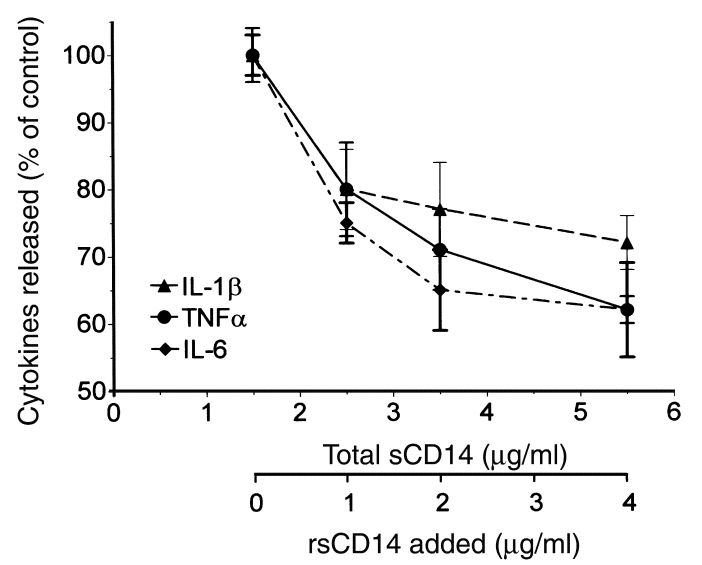

Figure 6

rsCD14 inhibits responses of LPS-loaded monocytes in whole human blood. Normal PBMCs were loaded with LPS by incubating the cells with LPS-sCD14 complexes ( 0.3 ng LPS per milliliter) for 5 minutes at $37^{\circ} \mathrm{C}$. The cells were washed to remove unbound LPS, and they were added to normal whole heparinized blood that contained the indicated concentrations of endogenous SCD14 and rsCD14. The mixtures were incubated for 5 hours, and cytokines (IL-1 $\beta$, TNF- $\alpha$, and IL-6) were measured in the plasma by ELISA. The cytokine levels are expressed as the percent of the mean level of cytokine released in blood that contained no rsCD14 (percent of control). Each point represents the mean \pm SEM of five or seven determinations from five independent experiments using blood from two normal donors.

rates of release of LPS from the cell surface. Given that both "on" and "off" rates of LPS-cell interactions should influence the net amount of LPS bound to the cells at any given time, we hypothesize that the increased apparent release rates observed in patient serum (Figure 3) decrease net LPS binding to the cells, even at the earliest time point as observed in severe sepsis serum (Figure 2b). Any increase in the rate of LPS-lipoprotein binding in the sepsis serum should also help decrease net LPS binding to the cells. However, our data do not rule out the possibility that serum from patients with severe sepsis also contains an inhibitor of LPS binding (Figure 2b).

Second, LPS efflux from monocytes, even in the plasma of sick patients, is regulated largely by soluble CD14 (Figure 5). This conclusion is consistent with our previous finding that $\mathrm{sCD} 14$ promotes LPS release from cells (12) and with the role of sCD14 in promoting phospholipid efflux from cells (21).

Third, sCD14 decreases monocyte responses to LPS. Although high blood levels of sCD14 have been associated with mortality in patients with Gram-negative sepsis (22), there is no evidence that sCD14 contributes to mortality. Injection of sCD14 decreased endotoxininduced mortality in a murine model (23). Depending on its concentration, sCD14 can either enhance or inhibit responses of cells that express mCD14 (24-26). Significant inhibition of in vitro responses was observed with sCD14 concentrations that were within the range observed in sepsis serum. Our data provide a mechanism for the inhibitory effect of sCD 14; sCD14 shifts the equilibrium between LPS-mCD14 and LPS-
sCD14 toward sCD14 (12), and increasing the sCD14 concentration also results in transfer of more cellbound LPS to lipoproteins. In keeping with these results, another study found that plasma sCD14 enhanced the sequestration of free LPS in plasma (27). In contrast, a recent report (28) showed that intracerebral injection of rsCD14 in a mouse model of bacterial meningitis increased cytokine levels in the cerebrospinal fluid. This raises the possibility that in extravascular compartments, where lipoprotein concentrations are lower, sCD14 can also have proinflammatory effects. The finding that increased levels of sCD14 in the serum of septic patients increase the response of an epithelial cell line to LPS (29) was reported as evidence of an inflammatory role for sCD14 in plasma. These experiments were performed with highly diluted serum and with cells that require sCD14 for activation because they do not express mCD14. Because we have found that even moderate dilution (1:3) of serum greatly increases LPS-monocyte interactions (data not shown), in vitro experiments using diluted serum may not accurately reflect the in vivo effects of the serum components.

We previously found that LBP plays little or no role in enhancing the release of monocyte-bound LPS (12). In experiments not shown, we found that the release of cell-bound LPS was not altered when we reduced LBP to normal levels by immunodepleting it from the serum of two septic patients. However, high LBP levels have been associated with decreased LPS responses in mice (30), so we cannot rule out the possibility that high LBP levels in humans might decrease cell responses to LPS by another mechanism.

The acute-phase response can alter both the levels and compositions of plasma lipoproteins (19). Numerous studies have shown that infection and acute illness lead to lower total cholesterol levels with a pronounced decline in HDL cholesterol $(19,20)$. We were therefore surprised to find that cell-associated LPS was sequestered by lipoproteins more rapidly in sepsis sera than in normal sera, which contain much more HDL. It is possible that the character, rather than the quantity, of the lipoprotein particles is a more important determinant of their ability to bind and sequester LPS. It is also possible that non-HDL lipoproteins become dominant acceptors during sepsis. These data cannot be explained entirely by elevated sCD14 levels. For example, we found that in normal serum, increasing the sCD14 concentration did not increase the fraction of the released LPS that bound to lipoproteins during a 10-minute incubation (see data in legend to Figure 5c); however, in serum from septic patients with elevated SCD14 levels, a greater fraction of the released LPS bound to lipoproteins during the same incubation period. Another component of sepsis plasma (either modified lipoproteins or another transfer protein) must work in concert with sCD14 to accelerate the binding of released LPS to lipoproteins. Efforts to identify this component are under way. 
Our data strongly suggests that both on and off events in LPS-monocyte interactions control cellular responses. In support of this hypothesis, we previously showed that plasma lipoproteins substantially reduce responses in monocytes that have already bound LPS (12), and we show here that responses are further reduced by sCD14. The actions of both lipoproteins and sCD14 in these assays appear to be LPS specific because they do not inhibit responses to IL-1 $\beta$. Reports from other laboratories are consistent with this hypothesis. Dedrick and Conlan (31) found that prolonged exposure of whole blood to LPS is required for maximal and prolonged cell responses. Other in vitro and in vivo studies have shown that LPS stimulation of leukocytes can be attenuated by agents that interfere with LPS-cell interactions even when they are administered after LPS. These include bactericidal permeability-increasing protein (31), Limulus antilipopolysaccharide factor (32), antibodies to CD14 (33), and reconstituted HDL (34).

During systemic responses to stress, blood leukocyte responses to LPS decrease. Although other mechanisms play very important roles in this attenuation, our results indicate that plasma can substantially reduce LPS-cell interactions. The ability of plasma constituents to regulate the amount and duration of LPS binding to cells suggests that these changes may help prevent systemic leukocyte activation during sepsis. Systemic leukocyte activation is generally thought to be detrimental to the host because it may provoke coagulopathy, organ failure, and shock. Controlling LPS-induced responses by circulating leukocytes may thus decrease systemic LPS toxicity while allowing inflammation to proceed at local tissue sites of microbial invasion.

\section{Acknowledgments}

We thank Peter Tobias for providing recombinant LPS binding protein, Leilani Bernardo for obtaining blood samples from ICU patients, and Borna Mehrad for critically reading the manuscript. This work was supported by NIH grants AI18188 and AI45896.

1. Kitchens, R.L. 2000. Role of CD14 in cellular recognition of bacterial lipopolysaccharides. In Chemical immunology: CD14 in the inflammatory response. Volume 74. R.S. Jack, editor. S. Karger AG. Basel, Switzerland. 61-82.

2. Poltorak, A., et al. 1998. Defective LPS signaling in $\mathrm{C} 3 \mathrm{H} / \mathrm{HeJ}$ and C57BL/10ScCr mice: mutations in Tlr4 gene. Science. 282:2085-2088.

3. Munford, R.S., Hall, C.L., Lipton, J.M., and Dietschy, J.M. 1982. Biological activity, lipoprotein-binding behavior, and in vivo disposition of extracted and native forms of Salmonella typhimurium lipopolysaccharides. J. Clin. Invest. 70:877-888.

4. Mathison, J.C., and Ulevitch, R.J. 1979. The clearance, tissue distribution, and cellular localization of intravenously injected lipopolysaccharide in rabbits. J. Immunol. 123:2133-2143.

5. Coulthard, M.G., Swindle, J., Munford, R.S., Gerard, R.D., and Meidell, R.S. 1996. Adenovirus-mediated transfer of a gene encoding acyloxyacyl hydrolase (AOAH) into mice increases tissue and plasma AOAH activity. Infect. Immun. 64:1510-1515.

6. Munford, R.S., Hall, C.L., and Dietschy, J.M. 1981. Binding of Salmonella typhimurium lipopolysaccharides to rat high-density lipoproteins. Infect. Immun. 34:835-843.

7. Parker, T.S., et al. 1995. Reconstituted high-density lipoprotein neutralizes gram- negative bacterial lipopolysaccharides in human whole blood.
Infect. Immun. 63:253-258.

8. Wurfel, M.M., Kunitake, S.T., Lichenstein, H., Kane, J.P., and Wright, S.D. 1994. Lipopolysaccharide (LPS)-binding protein is carried on lipoproteins and acts as a cofactor in the neutralization of LPS. J. Exp. Med. 180:1025-1035.

9. Vesy, C.J., Kitchens, R.L., Wolfbauer, G., Albers, J.J., and Munford, R.S. 1999. LPS binding protein and phospholipid transfer protein release lipopolysaccharides from gram negative bacterial membranes. Infect. Immun. 68:2410-2417.

10. Pajkrt, D., et al. 1996. Antiinflammatory effects of reconstituted high-density lipoprotein during human endotoxemia. J. Exp. Med. 184:1601-1608.

11. Read, T.E., et al. 1995. Triglyceride-rich lipoproteins prevent septic death in rats. J. Exp. Med. 182:267-272.

12. Kitchens, R.L., Wolfbauer, G., Albers, J.J., and Munford, R.S. 1999. Plasma lipoproteins promote the release of bacterial lipopolysaccharide from the monocyte cell surface. J. Biol. Chem. 274:34116-34122.

13. Kitchens, R.L., and Munford, R.S. 1998. CD14-dependent internalization of lipopolysaccharide (LPS) is strongly influenced by LPS aggregation but not by cellular responses to LPS. J. Immunol. 160:1920-1928.

14. Viriyakosol, S., Mathison, J.C., Tobias, P.S., and Kirkland, T.N. 2000. Structure-function analysis of CD14 as a soluble receptor for lipopolysaccharide. J. Biol. Chem. 275:3144-3149.

15. American College of Chest Physicians/Society of Critical Care Medicine Consensus Conference Committee. 1992. Definitions for sepsis and organ failure and guidelines for the use of innovative therapies in sepsis. Crit. Care Med. 20:864-874.

16. Munford, R.S., DeVeaux, L.C., Cronan, J.E., Jr., and Rick, P.D. 1992. Biosynthetic radiolabeling of bacterial lipopolysaccharide to high specific activity. J. Immunol. Methods. 148:115-120.

17. Kitchens, R.L., Thompson, P.A., O'Keefe, G.E., and Munford, R.S. 2000. Plasma constituents regulate LPS binding to, and release from, the monocyte cell surface. J. Endotoxin Res. 6:477-482.

18. Sahu, A., Kay, B.K., and Lambris, J.D. 1996. Inhibition of human complement by a C3-binding peptide isolated from a phage-displayed random peptide library. J. Immunol. 157:884-891.

19. Khovidhunkit, W., Memon, R.A., Feingold, K.R., and Grunfeld, C. 2000. Infection and inflammation-induced proatherogenic changes of lipoproteins. J. Infect. Dis. 181:462-472(Suppl.).

20. Gordon, B.R., et al. 1996. Low lipid concentrations in critical illness: implications for preventing and treating endotoxemia. Crit. Care Med. 24:584-589.

21. Sugiyama, T., and Wright, S.D. 2001. Soluble CD14 mediates efflux of phospholipids from cells. J. Immunol. 166:826-831.

22. Landmann, R., et al. 1995. Increased circulating soluble CD14 is associated with high mortality in gram-negative septic shock. J. Infect. Dis. 171:639-644.

23. Stelter, F., et al. 1998. Different efficacy of soluble CD14 treatment in high- and low-dose LPS models. Eur. J. Clin. Invest. 28:205-213.

24. Schütt, C., Schilling, T., and Kruger, C. 1991. sCD14 prevents endotoxin inducible oxidative burst response of human monocytes. Allerg. Immunol. (Leipz). 37:159-164.

25. Haziot, A., Rong, G.-W., Bazil, V., Silver, J., and Goyert, S.M. 1994. Recombinant soluble CD14 inhibits LPS-induced tumor necrosis factor- $\alpha$ production by cells in whole blood. J. Immunol. 152:5868-5876.

26. Hailman, E., et al. 1996. Stimulation of macrophages and neutrophils by complexes of lipopolysaccharide and soluble CD14. J. Immunol. 156:4384-4390.

27. Hiki, N., et al. 1999. Changes in endotoxin-binding proteins during major elective surgery: important role for soluble CD14 in regulation of biological activity of systemic endotoxin. Clin. Diagn. Lab. Immunol. 6:844-850.

28. Cauwels, A., et al. 1999. The origin and function of soluble CD14 in experimental bacterial meningitis. J. Immunol. 162:4762-4772.

29. Landmann, R., Reber, A.M., Sansano, S., and Zimmerli, W. 1996. Function of soluble CD14 in serum from patients with septic shock. J. Infect. Dis. 173:661-668.

30. Lamping, N., et al. 1998. LPS binding protein protects mice from septic shock caused by LPS or gram-negative bacteria. J. Clin. Invest. 101:2065-2071.

31. Dedrick, R.L., and Conlon, P.J. 1995. Prolonged expression of lipopolysaccharide (LPS)-induced inflammatory genes in whole blood requires continual exposure to LPS. Infect. Immun. 63:1362-1368.

32. Roth, R.I., Su, D.H., Child, A.H., Wainwright, N.R., and Levin, J. 1998. Limulus antilipopolysaccharide factor prevents mortality late in the course of endotoxemia. J. Infect. Dis. 177:388-394.

33. Schimke, J., Mathison, J., Morgiewicz, J., and Ulevitch, R.J. 1998. Anti$\mathrm{CD} 14 \mathrm{mAb}$ treatment provides therapeutic benefit after in vivo exposure to endotoxin. Proc. Natl. Acad. Sci. USA. 95:13875-13880.

34. Doran, J.E., Lerch, P., and Hubsch, A.P. 1994. Natural and artificial lipoproteins modulate lipopolysaccharide-induced tumour necrosis factor production in vitro and in vivo. Old Herborn University Seminar Monograph. 5:106-121. 